\title{
Intravenous citrulline generation test to assess intestinal function in intensive care unit patients
}

Job HC Peters'

Nicolette JWierdsma 2,3

Albertus Beishuizen ${ }^{4,5}$

Tom Teerlink ${ }^{6}$

\section{Ad $A$ van Bodegraven ${ }^{3,7}$}

'Department of Gastroenterology and Hepatology, Red Cross Hospital, Beverwijk, ${ }^{2}$ Department of Nutrition and Dietetics, VU University Medical Center, Amsterdam, ${ }^{3}$ Department of Gastroenterology, Small Bowel Disease Unit, VU University Medical Center, Amsterdam, ${ }^{4}$ Department of Intensive Care, VU University Medical Center, Amsterdam, ${ }^{5}$ Department of Intensive Care, Intensive Care Center, Medisch Spectrum Twente, Enschede, ${ }^{6}$ Department of Clinical Chemistry, Metabolic Laboratory, VU University Medical Center, Amsterdam, ${ }^{7}$ Department of Gastroenterology, Geriatrics, Intensive Care and Internal Medicin (Co-MIK), Zuyderland MC, HeerlenSittard-Geleen, the Netherlands

This article was published in the following Dove Press journal:

Clinical and Experimental Gastroenterology

28 April 2017

Number of times this article has been viewed

Background: Assessment of a quantifiable small intestinal function test is cumbersome. Fasting citrulline concentrations have been proposed as a measure of enterocyte function and elaborated into a citrulline generation test (CGT), which is applicable only when glutamine is administered orally. CGT is an oral test, limiting its use, for example, in critically ill patients.

Objective: Assessment of normative values and feasibility of an intravenously performed CGT in intensive care unit (ICU) patients with presumed gastrointestinal motility disturbances, especially when performed intravenously.

Design: CGT reference values were determined in 16 stable ICU patients using two different CGT methods, namely following either enteral or intravenous glutamine administration and both with simultaneous arterial and venous plasma citrulline sampling at six time-points. Plasma amino acid analysis was performed using reverse-phase high-performance liquid chromatography. Results: The median total generation of citrulline in 90 min (CGT iAUCT90) was markedly higher with arterial citrulline sampling compared with venous citrulline sampling, being $724 \pm 585$ and $556 \pm 418 \mu \mathrm{mol} / \mathrm{L} / \mathrm{min}$ for enteral glutamine, respectively $(p=0.02)$ and $977 \pm 283$ and $769 \pm 231 \mu \mathrm{mol} / \mathrm{L} / \mathrm{min}$ for intravenous glutamine, respectively $(p=0.0004)$. The median slope (time-dependent increase) for plasma arterial and venous citrulline during the CGT was $0.20 \pm 0.16$ and $0.18 \pm 0.12 \mu \mathrm{mol} / \mathrm{L} / \mathrm{min}$ for enteral glutamine, respectively $(p=0.004)$ and $0.22 \pm 0.16$ and $0.19 \pm 0.05 \mu \mathrm{mol} / \mathrm{L} / \mathrm{min}$ for intravenous glutamine, respectively $(p=0.02)$.

Conclusion: Intravenous glutamine administration combined with arterial plasma citrulline sampling yielded the least variation in CGT characteristics in stable ICU patients. A 2-point measurement test had comparable test characteristics as a 6-point measurement CGT and seems promising.

Keywords: citrulline, citrulline generation test, critical illness, enterocyte, intestinal function, intensive care, ICU, glutamine, HPLC

\section{Introduction}

Assessment of small intestinal (dys)function is cumbersome and difficult to quantify. ${ }^{1}$ In the last decades, citrulline has been proposed to assess the intestinal function by single measurement of a fasting concentration. ${ }^{2}$ Citrulline, which is a non-protein amino acid (not incorporated into proteins), is produced by small intestine from its precursor glutamine and is subsequently released into the systemic circulation, without

Correspondence: Nicolette J Wierdsma Department of Nutrition and Dietetics, VU University Medical Center, Amsterdam, the Netherlands $\mathrm{Tel}+3 \mathrm{I} 204443410$

Fax +3I 204444143

Email N.Wierdsma@vumc.nl appreciable metabolization by the liver. ${ }^{3-5}$ Citrulline is subsequently converted into arginine by kidney. Since the small bowel is by far the largest source of circulating citrulline, this amino acid is thought to be a suitable biomarker of enterocyte function. ${ }^{6}$ Recently, this mechanism has been questioned based on tracer studies in which 
the carbon skeleton of glutamine and not the amino nitrogen was followed. ${ }^{7,8}$ It has been proposed that citrulline might be generated by various pathways; however, an overdose of glutamine initiates a maximal generation of citrulline in a skewed bell-shaped curve. The clinical value of single fasting citrulline concentration measurement has been debated. ${ }^{9}$ Therefore, an enterocyte function test called the citrulline generation test (CGT) has been proposed several years ago. ${ }^{10}$ This test reflects the aforementioned physiologic pathway in which glutamine is converted into citrulline by the competent enterocyte (Figure 1). CGT implies the oral administration of a standardized amount of glutamine and subsequently the measurement of the increase in plasma citrulline which is generated by the enterocytes, enabling differentiation between healthy subjects and patients with reduced enterocyte mass. ${ }^{10}$

When oral administration of glutamine is precluded, for example, due to gastrointestinal motility disturbances, CGT cannot be performed. This may hamper the application of this oral test in majority of patients in an intensive care unit (ICU). Nevertheless, in ICU patients, adequate function of the gastrointestinal tract has been suggested to be of significant clinical relevance, especially with regard to nutritional support. ${ }^{11}$ In addition, the risk of systemic inflammation or sepsis may increase upon loss of the intestinal barrier function, which critically depends on an intact intestinal layer with functional enterocytes. ${ }^{12}$ However, no practical bedside tests are available to reliably measure small intestinal function in critically ill patients. Intestinal malabsorption in critically ill patients, which is more common than previously thought, ${ }^{13}$ is associated with worse clinical outcome. ${ }^{14-16}$ Therefore, practically applicable enterocyte function tests are warranted.

The primary aim of this study was to assess feasibility and reference CGT values in "stable" ICU patients. The secondary aim was to determine the differences in CGT characteristics between enteral and intravenous administration of glutamine, necessary in case of gastrointestinal motility disturbances,

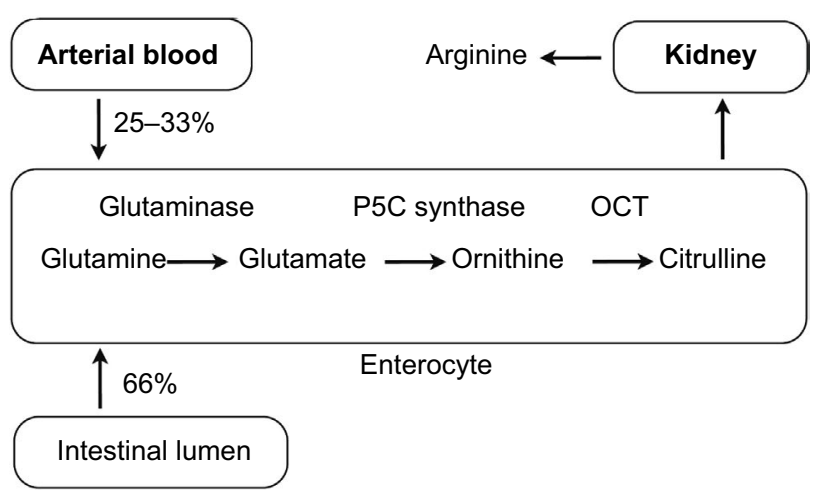

Figure I Physiologic pathway of glutamine into citrulline by the enterocyte. Abbreviations: $\mathrm{P5C}$, pyrroline-5-carboxylate; OCT, ornithine carbamoyltransferase. and to determine the differences in plasma citrulline concentrations in venous and arterial sampling. Finally, this study suggests to reduce the standard "6-point sampling" CGT to a simplified "2-point sampling" CGT in order to make it a more practical bedside test for future purpose.

\section{Materials and methods Patients}

In this observational cross-sectional study, a series of 16 "stable" ICU patients were included in a mixed medicosurgical ICU. Clinical characteristics of all the patients are summarized in Table 1. The definition of a "stable" ICU patient and the inclusion and exclusion criteria are summarized in Table 2. The ethics committee of the VU University Medical Center (Amsterdam, the Netherlands) approved the study protocol, and verbal informed consent was obtained from all the patients or their next of kin or other legal representative prior to inclusion.

Routine ICU data such as actual diagnosis, medical history, sex, age, body mass index, acute physiology and chronic health evaluation II (APACHE) and Sequential Organ Failure Assessment (SOFA) score, length of ICU stay, length of mechanical ventilation, medication record, route of enteral nutrition (nasogastric or nasojejunal), and daily and cumulative caloric intake provided by tube feeding were collected.

\section{The CGT}

Each ICU patient was submitted to one of the two different CGTs in a randomized fashion by using either enteral glutamine or intravenous glutamine, performed on separate days, after an overnight fast of at least $8 \mathrm{~h}$. During both the tests, simultaneous arterial and venous blood sampling was performed from either an indwelling arterial (radial) line or centra venous (subclavian or jugular) line. This led to CGT combinations:

- 1A: CGT following enteral glutamine administration, with arterial blood sampling.

- 1B: CGT following enteral glutamine administration, with venous blood sampling.

- 2A: CGT following intravenous glutamine administration, with arterial blood sampling.

- 2B: CGT following intravenous glutamine administration, with venous blood sampling

Baseline amino acid profiles and subsequent changes during the CGT were measured in blood samples obtained from indwelling arterial line or central venous lines. The central venous catheter was kept open in between blood collections by infusion of $\mathrm{NaCl} 0.9 \%$. The first $2 \mathrm{~mL}$ of 
Table I Basic characteristics of ICU patients included in this study

\begin{tabular}{|c|c|c|c|c|c|c|c|c|}
\hline Patient & $\begin{array}{l}\text { Age } \\
\text { (years) }\end{array}$ & Sex & $\begin{array}{l}\text { BMI } \\
\left(\mathrm{kg} / \mathrm{m}^{2}\right)\end{array}$ & $\begin{array}{l}\text { Daily intake } \\
\text { (kcal) }^{\mathrm{a}}\end{array}$ & Diagnosis & $\begin{array}{l}\text { APACHE } \\
\text { II score }\end{array}$ & $\begin{array}{l}\text { SOFA } \\
\text { score }^{c}\end{array}$ & Survival $^{\mathrm{d}}$ \\
\hline 1 & 67 & $\mathrm{~F}$ & 34.4 & 1943 & Type A aortic dissection & 26 & 4 & Alive \\
\hline 2 & 64 & M & 15.9 & 975 & ARF, pneumonia & 27 & 6 & Alive \\
\hline 3 & 64 & M & 31.1 & 1728 & Post-CABG & 21 & 3 & Alive \\
\hline 4 & 63 & $\mathrm{~F}$ & 44.1 & 3209 & Multiple myeloma & 16 & 3 & Alive \\
\hline 5 & 52 & $\mathrm{~F}$ & 23.4 & $140 \mid$ & ARF, COPD & 19 & 2 & Died \\
\hline 6 & 62 & $\mathrm{~F}$ & 23.1 & 1317 & Post-CABG & 28 & 4 & Alive \\
\hline 7 & 53 & M & 16.4 & 1203 & ARF, pneumonia & 27 & 5 & Alive \\
\hline 8 & 50 & M & 34.0 & 1784 & $\mathrm{OHCA}$ & 19 & 5 & Died \\
\hline 9 & 73 & $\mathrm{~F}$ & 31.1 & 1709 & $\mathrm{OHCA}$ & 39 & 4 & Alive \\
\hline 10 & 54 & $\mathrm{~F}$ & 26.3 & 1499 & Intracranial bleeding & 25 & 6 & Alive \\
\hline II & 71 & $\mathrm{~F}$ & 27.3 & 1554 & Type A aortic dissection & 26 & 3 & Alive \\
\hline 12 & 59 & $M$ & 23.3 & 1970 & ARF, pneumonia & 25 & 2 & Alive \\
\hline 13 & 41 & $\mathrm{~F}$ & 26.0 & 1750 & Type $A$ aortic dissection & 24 & 4 & Alive \\
\hline 14 & 68 & M & 23.5 & 1712 & ARF, pulmonary embolism & 24 & I & Alive \\
\hline 15 & 54 & $\mathrm{~F}$ & 22.9 & 1803 & ARF, intoxication sedatives & 19 & 3 & Alive \\
\hline 16 & 79 & $M$ & 23.9 & 1047 & ARF, pneumonia & 24 & 4 & Alive \\
\hline \multirow[t]{2}{*}{ Mean \pm SD } & $60.9 \pm 9.8$ & $9 \mathrm{~F}$ & $26.7 \pm 7.0$ & $1663 \pm 510$ & & $24.3 \pm 5.3$ & $3.7 \pm 1.4$ & 2 Died \\
\hline & & $7 M$ & & & & & & I4 Alive \\
\hline
\end{tabular}

Notes: a Mean caloric intake in kcal $24 \mathrm{~h}$ prior to the first CGT (corrected for $8 \mathrm{~h}$ fasting period); ${ }^{\mathrm{b}}$ score indicating first $24 \mathrm{~h}$ after admission, not during CGT; 'SOFA score at day of first CGT; 30 days mortality.

Abbreviations: ICU, intensive care unit; BMI, body mass index; CGT, citrulline generation test; F, female; M, male; ARF, acute respiratory failure; CABG, coronary artery bypass grafting; OHCA, out of hospital cardiac arrest; APACHE, acute physiology and chronic health evaluation; SOFA, Sequential Organ Failure Assessment.

Table 2 Inclusion and exclusion criteria for CGT study in ICU patients

Inclusion criteria

- Age between 18 and 80 years

- Verbal informed consent

- Fecal output $<250 \mathrm{~mL}$ per day

Definition of a "stable" ICU patient

I. Any ICU patient admitted for mechanical ventilation, with stable hemodynamics as judged by the intensivist and an expected ICU stay of $>72 \mathrm{~h}$

2. Ability to tolerate enteral nutrition, provided by either nasogastric or nasojejunal feeding tube, meeting full protein energy requirements based on indirect calorimetric measurements and further individualized according to an automated nutritional program ${ }^{23}$

\section{Exclusion criteria}

- Documented causes of small bowel malabsorption possibly interfering with intestinal absorptive function, eg, coeliac disease, Crohn's disease of the small bowel, exocrine pancreatic insufficiency, radiation enteritis, or short bowel syndrome

- Small intestinal resections and/or extensive colonic resection $(>2 / 3)$

- Liver cirrhosis Child-Pugh B or C, acute liver failure or renal failure requiring artificial support (eg, CVVH)

- Urea cycle defects/citrullinemia

- Exogenous steroids in excess of $10 \mathrm{mg}$ prednisolone equivalent (leading to P5C synthase induction leading to increased production of citrulline from glutamine in the enterocytes)

- Pregnancy (no long-term data are available on the effects of Dipeptiven ${ }^{\circledR}$ during pregnancy or lactation)

- Use of (par)enteral glutamine/citrulline supplements

Abbreviations: CGT, citrulline generation test; ICU, intensive care unit; CVVH, continuous veno-venous hemofiltration; P5C, pyrroline-5-carboxylate.

blood was excluded from sampling analysis to prevent dilution with $\mathrm{NaCl} 0.9 \%$.

\section{CGT with enteral administered glutamine}

At baseline, $100 \mathrm{~mL}$ of Dipeptiven ${ }^{\circledR}$ (Fresenius Kabi, Bad Homburg, Germany) containing $20 \mathrm{~g}$ of the dipeptide alanine-glutamine (ie, $13.46 \mathrm{~g}$ of 1-glutamine) was given by nasogastric or nasojejunal feeding tube. Subsequently, the tube was flushed with $10 \mathrm{~mL}$ of $\mathrm{NaCl} 0.9 \%$. Blood samples for amino acid analysis were obtained at baseline, 20, 40, 60, $75,90,120,150$, and $180 \mathrm{~min}$.

\section{CGT with intravenously administered glutamine}

At baseline, $300 \mathrm{~mL}$ of distilled water was added to $100 \mathrm{~mL}$ of Dipeptiven ${ }^{\circledR}$, to reduce osmolarity of the solution, and was subsequently administered intravenously over a period of $30 \mathrm{~min}$. Blood samples for amino acid analysis were obtained at baseline, 20, 40, 60, 75, 90, 120, 150, and $180 \mathrm{~min}$. 


\section{Assessment of plasma amino acid concentrations}

Venous and arterial blood samples taken during the CGT were collected in heparin tubes and directly placed on ice. After centrifugation $\left(10 \mathrm{~min}\right.$ at $3000 \mathrm{rpm}$ and $\left.18^{\circ} \mathrm{C}\right)$, $500 \mu \mathrm{L}$ of plasma was poured in to a cryovial containing $20 \mathrm{mg}$ of dry sulfosalicylic acid for de-proteinizing. Then, the cryovial was vortexed and stored at $-80^{\circ} \mathrm{C}$ until analysis. The concentrations of citrulline, glutamine, alanine, and arginine were determined using reverse-phase highperformance liquid chromatography as previously described by Teerlink et al. ${ }^{17}$

\section{CGT variables "slope" and "iAUCT90"}

As documented earlier in the proof of principle study on CGT, ${ }^{8}$ the slope of the line between the baseline plasma citrulline concentration and peak plasma citrulline level during the CGT was calculated, reflecting the ability of citrulline generation from administered glutamine by the enterocytes. Then, the incremental area under the CGT curve was measured from 0 to $90 \mathrm{~min}$ (iAUCT90; incremental area under the plasma citrulline concentration vs time curve). ${ }^{10}$

\section{Statistical analysis}

Data are expressed as mean \pm standard deviation (SD). Two-way analysis of variance (ANOVA) was performed while comparing slope and iAUCT90 between various CGT combinations. When a statistically significant difference among groups was encountered by using ANOVA, a Tukey's multiple comparison test was performed. Statistical significance was accepted at $p<0.05$. Variance analysis between various CGTs was performed using Bartlett's test. To compare the differences between arterial or venous blood sampling during CGT and enteral or intravenous glutamine administration, a 2-sided paired $t$-test was performed. Association tests were performed by means of Pearson's correlation test. GraphPad Prism 4.0c (GraphPad Software, Inc., La Jolla, CA, USA) for Macintosh and EXCEL were used to perform statistical analysis and to design the figures shown in this study.

\section{Results}

CGT characteristics in the 16 stable ICU patients were obtained according to protocol. Figure 2 depicts the mean plasma citrulline changes during various CGT methods. Figure 3 shows the CGT iAUCT90 for every individual patient for all CGT variants.

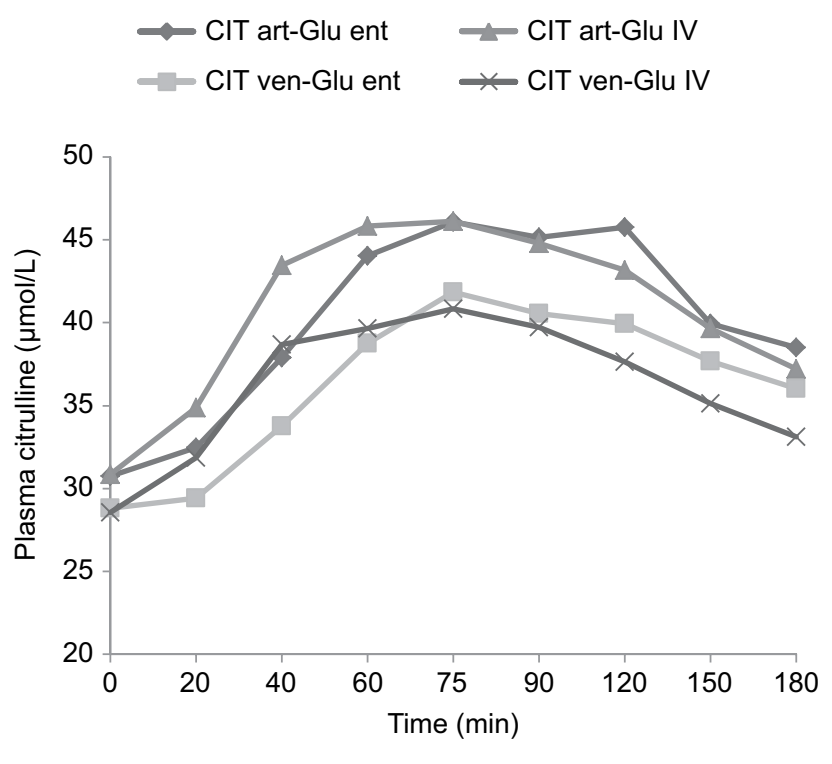

Figure 2 Mean plasma citrulline changes of various citrulline generation test methods (enteral-intravenous, arterial-venous) in 16 stable intensive care unit patients.

Abbreviations: Cit, citrulline; art, arterial; IV, intravenous; Glu, glutamine; ent, enteral; ven, venous.

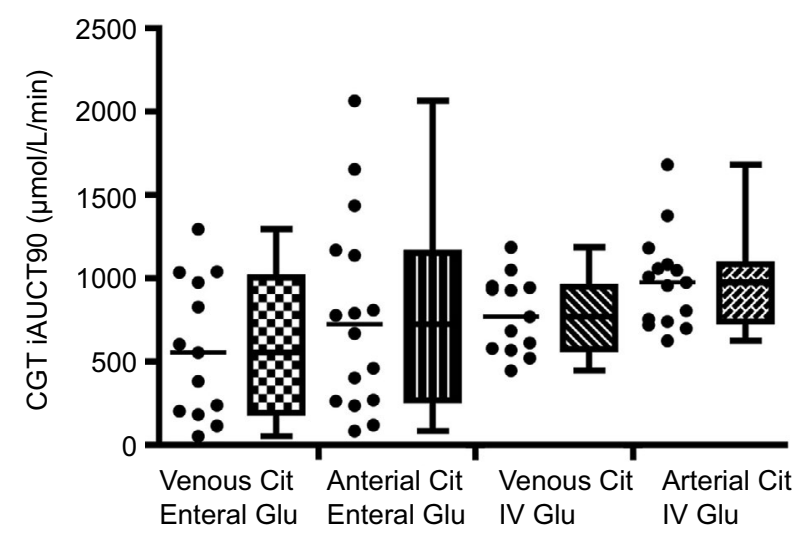

Figure 3 Incremental area under the curve after $90 \mathrm{~min}$ (iAUCT90) of various CGT methods (enteral-intravenous, arterial-venous) in 16 stable intensive care unit patients. Dots represent individual values versus box and whisker plot.

Abbreviations: CGT, citrulline generation test; Cit, citrulline; IV, intravenous; Glu, glutamine.

\section{CGT using intravenous versus enteral glutamine administration}

There was no statistically significant difference in mean baseline plasma citrulline concentration and maximum plasma citrulline concentration after either intravenous or enteral glutamine administration (see Table 3). Mean time to peak citrulline during the CGT was comparable when glutamine was given intravenously or enterally, being $72 \pm 24$ and $94 \pm 37$ min with arterial blood sampling, respectively $(p=0.07)$ and $82 \pm 20$ and $99 \pm 39$ min with venous blood sampling, respectively $(p=0.21)$. 
Table 3 CGT reference values (mean \pm SD) based on different methodologies in stable ICU patients $(n=16)$

\begin{tabular}{|c|c|c|c|c|}
\hline \multirow[t]{2}{*}{ Citrulline sampling } & \multicolumn{2}{|c|}{ CGT I enteral glutamine } & \multicolumn{2}{|c|}{ CGT 2 IV glutamine } \\
\hline & Venous & Arterial & Venous & Arterial \\
\hline Baseline citrulline $(\mu \mathrm{mol} / \mathrm{L})$ & $29 \pm 7$ & $31 \pm 8$ & $29 \pm 9$ & $31 \pm 9$ \\
\hline Peak citrulline $(\mu \mathrm{mol} / \mathrm{L})$ & $45 \pm 14$ & $51 \pm 16$ & $42 \pm 12$ & $48 \pm 14$ \\
\hline Time to peak (min) & $99 \pm 39$ & $94 \pm 37$ & $82 \pm 20$ & $72 \pm 24$ \\
\hline Slope ${ }^{a}$ & $0.18 \pm 0.12^{*}$ & $0.24 \pm 0.16^{*}$ & $0.19 \pm 0.05 *$ & $0.28 \pm 0.16^{*}$ \\
\hline iAUCT90 $(\mu \mathrm{mol} / \mathrm{L} / \mathrm{min})^{\mathrm{b}}$ & $578 \pm 418^{*}$ & $772 \pm 585^{*}$ & $784 \pm 231 *$ & $982 \pm 283 *$ \\
\hline
\end{tabular}

Notes: a Slope from baseline to peak plasma citrulline concentration during CGT. Incremental area under the CGT curve between 0 and 90 min. ${ }^{*} p<0.05$ (arterial vs venous within either CGT I or 2).

Abbreviations: CGT, citrulline generation test; SD, standard deviation; ICU, intensive care unit; IV, intravenous; iAUCT90, incremental area under curve after 90 min.

There was no statistically significant difference in mean slope for plasma citrulline following intravenous or enteral glutamine administration (for both arterial and venous blood sampling). There was a statistical consistent trend for an increased mean CGT iAUCT90 following intravenous glutamine with arterial blood sampling, being $982 \pm 283$ and $772 \pm 585 \mu \mathrm{mol} / \mathrm{L} / \mathrm{min}$ for intravenous and enteral glutamine, respectively $(p=0.09)$. The same applied to the mean CGT iAUCT90 using venous blood sampling, being $784 \pm 231$ and $578 \pm 418 \mu \mathrm{mol} / \mathrm{L} / \mathrm{min}$ for intravenous and enteral glutamine, respectively $(p=0.07)$.

\section{CGT using arterial versus venous blood sampling}

There were no differences in baseline plasma citrulline concentration, maximum plasma citrulline concentration, and time to peak citrulline using either arterial or venous blood sampling (Table 3).

The mean slope for plasma citrulline during the CGT was steeper when blood was sampled arterially compared with venous sampling, being $0.24 \pm 0.16$ and $0.18 \pm 0.12$ $\mu \mathrm{mol} / \mathrm{L} / \mathrm{min}$ for enteral glutamine, respectively $(p=0.004)$ and $0.28 \pm 0.16$ and $0.19 \pm 0.05 \mu \mathrm{mol} / \mathrm{L} / \mathrm{min}$ for intravenous glutamine, successively $(p=0.02)$. The mean CGT iAUCT 90 was also increased with arterial sampling compared to venous blood sampling, being $772 \pm 585$ and $578 \pm 418 \mu \mathrm{mol} / \mathrm{L} / \mathrm{min}$ for enteral glutamine, respectively $(p=0.02)$, and $982 \pm 283$ and $784 \pm 231 \mu \mathrm{mol} / \mathrm{L} / \mathrm{min}$ for intravenous glutamine, successively $(p=0.0004)$.

\section{Variation between different CGT methods}

The coefficient of variation for the iAUCT90s in the four different CGTs as shown in Figure 3 was $76 \%$ for enteral administered glutamine combined with arterial citrulline sampling, $72 \%$ for enteral glutamine with venous citrulline sampling, $29 \%$ for intravenous glutamine with arterial

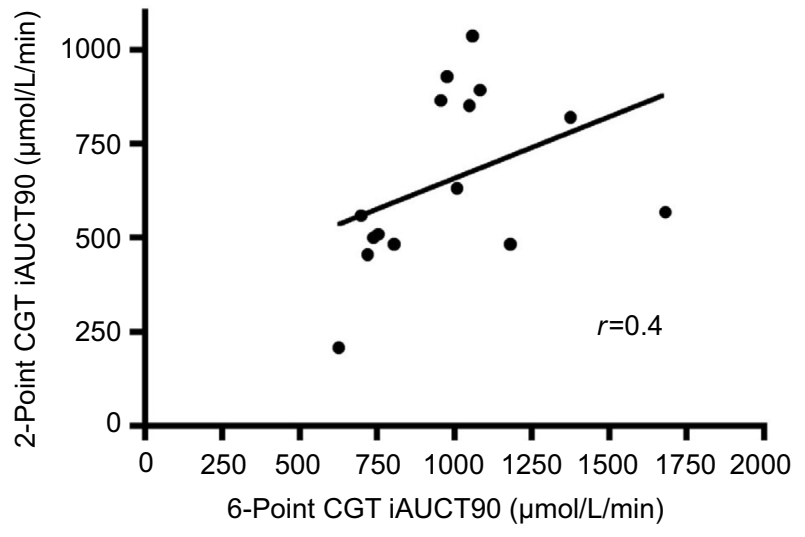

Figure 4 Correlation between the standard incremental area under curve after 90 min (iAUCT90; intravenous glutamine administration and arterial sampling) of citrulline generation test (CGT) and the simplified 2-point CGT in 16 stable intensive care unit patients.

citrulline measurement, and 30\% for intravenous glutamine with venous citrulline measurement.

\section{Reducing number of blood samples during CGT}

Figure 4 shows the correlation between the standard CGT iAUCT90 (using arterial blood sampling and intravenous glutamine administration) consisting of six blood samples $(0,20$, $40,60,75$, and $90 \mathrm{~min}$ ) and a simplified 2-point variation in which the iAUC was calculated including only the first and last blood sample ( 0 and $90 \mathrm{~min}$ ) used in the standard CGT (Pearson's $r=0.4$ ). Variation between individual values (as shown in Figure 3) was 35\% for the 2-point CGT iAUCT90 compared with $29 \%$ for the standard 6-point CGT iAUCT 90.

\section{Discussion}

This study was performed to explore the variations of a previously proposed oral enterocyte function test, CGT, in order to determine the reference values of enterally or intravenously applied glutamine and to compare different CGT methods. It was found that the "standard" CGT using enteral glutamine seemed to be suboptimal in a population of stable but by 
nature complex ICU patients. When the citrulline precursor glutamine was provided through the enteral route, large variation in CGT parameters, for example, the CGT slope and iAUCT90, was observed. The variation in citrulline CGT slope and iAUCT90 greatly diminished when glutamine was intravenously given in the same patients. It was assumed that decreased gastrointestinal motility (ie, a prolonged gastrointestinal transit time, which is a common finding in ICU patients) was the main determinant of the variation in CGT outcomes in ICU patients receiving enteral glutamine, more than differences in enterocyte mass.

Of additional interest was that variation in CGT outcome between enteral and intravenous glutamine could not be explained by nasogastric or nasojejunal feeding route (data not shown). Rauch et al also observed a high variation in gastrointestinal transit time using video capsule endoscopy in 16 neurosurgical critically ill patients without prior documented intestinal impairment. ${ }^{18}$ Also, one could theorize that glutamine given intravenously may reach more of the small intestine compared with glutamine administered enterally, which may only reflect proximal small intestinal citrulline production.

Arterial blood sampling resulted in statistically significantly higher citrulline concentrations compared with venous blood sampling, irrespective of the route of glutamine administration (intravenous or enteral). In a human tracer study, the net hepatic flux of citrulline was negligible. ${ }^{19}$ Hence, the higher arterial citrulline levels during the CGT compared with the concomitant venous sampling could not be explained by hepatic interference with citrulline metabolism.

The CGT has originally been developed for the assessment of intestinal function in healthy individuals without gastrointestinal disorders after oral administration of a fixed dose of glutamine. ${ }^{10}$ Current results in stable ICU patients were essentially similar to those observed in healthy controls (these comprised as reference value; a baseline plasma citrulline concentration of $38 \pm 8 \mu \mathrm{mol} / \mathrm{L}$ ); a peak value of $55 \pm 10 \mu \mathrm{mol} / \mathrm{L}$; a calculated slope of $0.22 \pm 0.08, \mu \mathrm{mol} / \mathrm{L} / \mathrm{min}$; a time to peak of $77 \pm 16 \mathrm{~min}$; an increment of $44 \pm 13 \%$, and, finally, an AUCT90 of $632 \pm 222 \mu \mathrm{mol} / \mathrm{L})$. Together with the absence of signs of diarrhea, a previously shown biomarker of malabsorption, ${ }^{10}$ it was presumed that the studied population of stable ICU patients have normal gastrointestinal function (as expected at forehand). The presented reference or normative values, based on mean data in the different test methodologies, may be applied in diseased conditions such as in short bowel syndrome, celiac disease or multi organ dysfunction syndrome (MODS) patients, the latter in which only parenteral administration of test substance is reproducibly feasible. Apart from the small intestine, kidney is also a determinant of plasma citrulline concentrations. The currently studied population all had normal renal function (data not shown). In stable isotope studies, it has been shown that renal conversion of plasma citrulline to arginine was $76 \pm 10 \%$ of plasma turnover of citrulline..$^{20,21}$ In the present study, plasma arginine changes when different CGT methods were compared. Arterial plasma arginine levels were lower than the venous plasma arginine concentrations (data not shown). These kinetic findings combined may indicate that the arterial-venous plasma citrulline difference during the CGT originated from renal conversion of citrulline into arginine.

Finally, this study explored the option of reducing the frequency of blood sampling during the CGT from six to two blood samples. According to the current CGT protocol, six separate blood samples between 0 and 90 min were required. ${ }^{10}$ Simplifying the sampling protocol without reducing the test accuracy was considered of practical importance to implement future bedside application of the CGT. Although the correlation between the standard 6-point CGT and the simplified 2-point CGT showed a relatively low correlation $(r=0.4)$, this outcome was skewed by the three highest 6-point iAUCT90 values (Figure 4). Excluding these three outliers increased the correlation ( $r=0.9$, data not shown). In terms of variation for each individual iAUCT T90 value, the 2-point test performed similarly (35\%) compared with the 6-point test $(29 \%)$. This study suggests that the 2-point test may be applicable in clinical setting; however, future studies to corroborate the clinical applicability and value of a 2-point CGT seem warranted.

Limitation of this study was the relatively heterogeneous ICU population, however without clinical signs of malabsorption. ${ }^{22}$ In addition, CGT outcomes were not correlated with any clinical and outcome parameters. The relationship between the CGT and other tests for intestinal function was not studied, as no gold standard test to assess the intestinal function in general or in critically ill patients exists.

In conclusion, CGT reference values (intravenous) in stable ICU patients with clinically normal liver, kidney, and (metabolic) intestinal function were determined. Of the different CGT methods tested, intravenous glutamine administration combined with arterial plasma citrulline sampling yielded the least variation in CGT characteristics. A proposal for a simplified 2-point CGT seemed comparable in relevant test characteristics and therefore clinically promising. The proposed intravenous CGT opens venues for further research on (ICU) patients with gastrointestinal motility disorders. 


\section{Acknowledgments}

The authors would like to thank Sigrid de Jong from the Department of Clinical Chemistry of the VU University Medical Center for performing the amino acid analysis and ICU research nurses Ingrid van den Hul and Erna Alberts for their pivotal logistic support.

\section{Author contributions}

$\mathrm{AB}, \mathrm{JHCP}, \mathrm{NJW}$, and AAvB designed the protocol. AB, JHCP, and $A A v B$ conducted the study and performed statistical analysis. TT provided essential materials. AB, JHCP, and AAvB wrote the first draft of the paper, and NJW, AAvB, and $A B$ had primary responsibility for the final content. All authors contributed toward data analysis, drafting and revising the paper and agree to be accountable for all aspects of the work.

\section{Disclosure}

The authors report no conflicts of interest in this work.

\section{References}

1. Peters JH, Beishuizen A, Keur MB, Dobrowolski L, Wierdsma NJ van Bodegraven AA. Assessment of small bowel function in critical illness: potential role of citrulline metabolism. J Int Care Med. 2011; 26(2):105-110.

2. Crenn P, Messing B, Cynober L. Citrulline as a biomarker of intestinal failure due to enterocyte mass reduction. Clin Nutr. 2008;27(3):328-339.

3. Windmueller HG, Spaeth AE. Source and fate of circulating citrulline. Am J Physiol. 1981;241:E473-E480.

4. Wakabayashi Y, Jones ME. Pyrroline-5-carboxylate synthesis from glutamate by rat intestinal mucosa. J Biol Chem. 1983;258:3865-3872.

5. Wu G. Intestinal mucosal amino acid catabolism. J Nutr. 1998;128: 1249-1252.

6. Crenn P, Coudray-Lucas C, Thuillier F, Cynober L, Messing B. Postabsorptive plasma citrulline concentration is a marker of absorptive enterocyte mass and intestinal failure in humans. Gastroenterology. 2000;119:1496-1505.

7. Ligthart-Melis GC, Marini JC, Engelen MP, Deutz NE. Glutamine supplementation, citrulline production, and de novo arginine synthesis: is there a relation? Am J Clin Nutr. 2015;101(4):890-892.

8. Marini JC. Interrelationships between glutamine and citrulline metabolism. Curr Opin Clin Nutr Metab Care. 2016;19(1):62-66.
9. Peters JH, Wierdsma NJ, Teerlink T, van Leeuwen PAM, Mulder CJ, van Bodegraven AA. Poor diagnostic accuracy of a single fasting plasma citrulline concentration to assess intestinal energy absorption capacity. Am J Gastro. 2007;102(12):2814-2819.

10. Peters JH, Wierdsma NJ, Teerlink T, van Leeuwen PAM, Mulder CJJ, van Bodegraven AA. The citrulline generation test: proposal for a new enterocyte function test. Aliment Pharmacol Ther. 2008;27(12):1300-1310.

11. Blackburn GL, Wollner S, Bistrian BR. Nutrition support in the intensive care unit: an evolving science. Arch Surg. 2010;145:533-538.

12. Gatt M, Reddy BS, MacFie J. Review article: bacterial translocation in the critically ill--evidence and methods of prevention. Aliment Pharmacol Ther. 2007;25:741-757.

13. Strack van Schijndel RJ, Wierdsma NJ, et al. Fecal energy losses in enterally fed intensive care patients: an explorative study using bomb calorimetry. Clin Nutr. 2006;25:758-764.

14. Sungurtekin H, Sungurtekin U, Oner O, Okke D. Nutrition assessment in critically ill patients. Nutr Clin Pract. 2008;23:635-641.

15. Doig CJ, Sutherland LR, Sandham JD, Fick GH, Verhoef M, Meddings JB. Increased intestinal permeability is associated with the development of multiple organ dysfunction syndrome in critically ill ICU patients. Am J Respir Crit Care Med. 1998;158:444-451.

16. McClave SA, Martindale RG, Vanek VW, et al. Guidelines for the provision and assessment of nutrition support therapy in the adult critically ill patient: Society of Critical Care Medicine (SCCM) and American Society for Parenteral and Enteral Nutrition (A.S.P.E.N.). JPEN J Parenter Enteral Nutr. 2009;33:277-316.

17. Teerlink T, van Leeuwen PA, HoudijkA. Plasma amino acids determined by liquid chromatography within 17 minutes. Clin Chem. 1994;40:245-249.

18. Rauch S, Krueger K, Turan A, Roewer N, Sessler DI. Determining small intestinal transit time and pathomorphology in critically ill patients using video capsule technology. Intensive Care Med. 2009;35:1054-1059.

19. Van de Poll MC, Ligthart-Melis GC, Boelens PG, Deutz NE, van Leeuwen PA, Dejong CH. Intestinal and hepatic metabolism of glutamine and citrulline in humans. J Physiol. 2007;581:819-827.

20. Ligthart-Melis GC, van de Poll MC, Boelens PG, Dejong CH, Deutz NE, van Leeuwen PA. Glutamine is an important precursor for de novo synthesis of arginine in humans. Am J Clin Nutr. 2008;87:1282-1289.

21. Buijs N, Brinkmann SJ, Oosterink JE, et al. Intravenous glutamine supplementation enhances renal de novo arginine synthesis in humans: a stable isotope study. Am J Clin Nutr. 2014;100(5):1385-1391.

22. Wierdsma NJ, Peters JH, Weijs PJ, et al. Malabsorption and nutritional balance in the ICU-faecal weight as a biomarker: a prospective observational pilot study. Crit Care. 2011;15(6):R264.

23. Strack van Schijndel RJ, Weijs PJ, Koopmans RH, Sauerwein HP, Beishuizen A, Girbes AR. Optimal nutrition during the period of mechanical ventilation decreases mortality in critically ill, long-term acute female patients: a prospective observational cohort study. Crit Care. 2009; 13:R132.
Clinical and Experimental Gastroenterology

\section{Publish your work in this journal}

Clinical and Experimental Gastroenterology is an international, peerreviewed, open access, online journal publishing original research, reports, editorials, reviews and commentaries on all aspects of gastroenterology in the clinic and laboratory. This journal is included on PubMed. The manuscript management system is completely online

\section{Dovepress}

and includes a very quick and fair peer-review system, which is all easy to use. Visit http://www.dovepress.com/testimonials.php to read real quotes from published authors. 